\title{
Simulation study for magnetic levitation in pure water exploiting the ultra-high magnetic field gradient product of a Hybrid Trapped Field Magnet Lens (HTFML)
}

K Takahashi ${ }^{1}$, H Fujishiro ${ }^{1}$ and M. D. Ainslie ${ }^{2}$

1) Department of Physical Science and Materials Engineering, Faculty of Science and Engineering, Iwate University, Morioka 020-8551, Japan

${ }^{2)}$ Bulk Superconductivity Group, Department of Engineering, University of Cambridge, Cambridge CB2 1PZ, UK

E-mail: s3119004@iwate-u.ac.jp, fujishiro@iwate-u.ac.jp, mark.ainslie@eng.cam.ac.uk

\section{$\underline{\text { Abstract }}$}

A hybrid trapped field magnet lens (HTFML) is a promising device that is able to concentrate a magnetic field higher than the applied field continuously, even after removing an external field, which was conceptually proposed by the authors in 2018. In this study, we propose a new additional advantage of the HTFML, which could be applicable for magnetic levitation and separation. The HTFML device consisting of $\mathrm{GdBaCuO}$ bulk cylinder and $\mathrm{GdBaCuO}$ magnetic lens, after the magnetization process from an applied field, $B_{\text {app }}=10 \mathrm{~T}$, can generate a maximum trapped field, $B_{\mathrm{c}}$ $=11.4 \mathrm{~T}$, as well as an ultra-high magnetic field gradient product, $B_{\mathrm{Z}} \cdot d B_{\mathrm{Z}} / d z$, over $\pm 3,000 \mathrm{~T}^{2} / \mathrm{m}$ at $T_{\mathrm{s}}$ $=20 \mathrm{~K}$, which is higher than that of existing superconducting magnets (SM) and large-scale hybrid magnets (HM). Through detailed numerical simulations, the HTFML device is considered for magnetic separation of a mixture of precious metal particles ( $\mathrm{Pt}, \mathrm{Au}, \mathrm{Ag}$ and $\mathrm{Cu}$ ) dispersed in pure water, by exploiting the magneto-Archimedes effect. The HTFML can be realized as a compact and mobile desktop-type superconducting bulk magnet system and there are a wide range of potential industrial applications, such as in the food and medical industries.

\section{Introduction}

The realization of superconducting magnets that can generate stationary magnetic fields up to 40 tesla has been encouraged by many types of fundamental studies related to magnetism: magnetic levitation [1-2] and separation [3-4], crystal growth [5-6] and nuclear magnetic resonance (NMR) [78]. The magnetic levitation technique, in particular, needs a strong magnetic field from an external magnet to realize the counter-balanced levitated situation against gravity acting on a diamagnetic material. The technique was first demonstrated for a diamagnetic water drop, in which the magnetic field gradient product, $B_{\mathrm{z}} \cdot d B_{\mathrm{z}} / d z$, was required to be as large as $-1400 \mathrm{~T}^{2} / \mathrm{m}$ along the upper direction 
of the vertical magnet [9]. Such a large $B_{\mathrm{z}} \cdot d B_{\mathrm{z}} / d z$ value is required because of the low magnetic susceptibility of water and is difficult to achieve with a conventional superconducting magnet. Thus, up to now, such magnetic levitation has been realized only by using a comparatively large-scale hybrid magnet in a specialized facility [10]. In the past, such large-scale hybrid magnets have been demonstrated with $B_{\mathrm{z}} \cdot d B_{\mathrm{z}} / d z$ values around $-1500 \mathrm{~T}^{2} / \mathrm{m}$ or better [11-12]. The $B_{\mathrm{Z}} \cdot d B_{\mathrm{Z}} / d z$ value for a standard $10 \mathrm{~T}$ cryo-cooled superconducting magnet is much lower, up to $-500 \mathrm{~T}^{2} / \mathrm{m}$, which has been enhanced with a ferromagnetic iron yoke up to $-1060 \mathrm{~T}^{2} / \mathrm{m}$ [2]. The magneto-Archimedes technique, proposed in 1998, also enables the reduction of the required $B_{\mathrm{z}} \cdot d B_{\mathrm{z}} / d z$ for the magnetic levitation of any target objects in a paramagnetic medium gas or liquid (e.g., oxygen or aqueous $\mathrm{MnCl}_{2}$ ) [13]. This method is utilized for the magnetic separation system for structural isomers of phthalic acid using oxygen and fluorocarbon instead of $\mathrm{MnCl}_{2}$ solution, as introduced in [3]. In such magnetic applications, the whole system, including the magnetic source, separation unit and medium solution, must be considered, based on the physical properties of the target objects. It would be also desirable to avoid using any harmful materials, as well as to construct the separation system in a simple way for ease-of-processing, reliability and stability. The intensity of the magnetic force, $F_{\mathrm{m}}$, is potentially restricted by the performance of the magnet used; however, it is not particularly cost-effective and realistic to build a conventional superconducting magnet or a large-scale magnet in any industrial facilities or laboratories, except for experimental purposes [6]. There is still a demand for a new magnetic field source that can provide both a stronger magnetic field, $B_{\mathrm{z}}$, and magnetic field gradient product, $B_{\mathrm{z}} \cdot d B_{\mathrm{z}} / d z$, if it can be done in a cost-effective and efficient way.

Large, single-grain (RE)BaCuO bulk superconductors (RE: rare earth element or $\mathrm{Y}$ ) can trap or expel magnetic flux, owing to the induced supercurrent flowing inside the material with zero resistance, and that functionality depends on the magnetizing method. Towards practical engineering applications, research advances in bulk superconductivity, including the relevant technologies such as material processing, cryogenic systems and magnetization techniques, are summarized in [14].

Such superconducting bulks, magnetized by field-cooled magnetization (FCM) as a so-called trapped field magnet (TFM), can trap magnetic flux inside the bulk due to the "vortex pinning effect" and generate quasi-permanent magnetic fields over $17 \mathrm{~T}$ at magnetizing temperatures below $30 \mathrm{~K}$. A record-high trapped field of 17.6 T has been reliably achieved by FCM at $26 \mathrm{~K}$ in the gap between two $\mathrm{GdBaCuO}$ disk bulks of $25 \mathrm{~mm}$ in diameter [15-16]. Pulsed-field magnetization (PFM) is also known as a key magnetizing technique to develop a desktop and mobile magnetizing system, for which the dynamic magnetic flux behavior and the resultant heat generation inside the bulk have been carefully investigated to improve trapped fields using PFM [17-19]. The record-high trapped field by PFM is $5.2 \mathrm{~T}$ at $28 \mathrm{~K}$ on the surface of a $45 \mathrm{~mm}$ diameter $\mathrm{GdBaCuO}$ disk bulk [20], and $5.3 \mathrm{~T}$ at 30 $\mathrm{K}$ in the gap between two $25 \mathrm{~mm}$ diameter $\mathrm{GdBaCuO}$ disk bulks [21]. In this way, bulk superconductors have been considered as a strong and compact magnetic field source, which could 
realize a desktop-type mobile magnet for application in a cryogen-free NMR system [22-23] and Lorentz force velocimetry (LFV) [24].

Based on the critical state model by Bean [25-26], the trapped field at the center of the top surface of a disc-shaped bulk, $B_{\mathrm{T}}$, can be expressed as

$$
B_{T} \approx k \mu_{0} J_{c} d,
$$

where $\mu_{0}$ is the permeability of free space, $J_{\mathrm{c}}$ is the critical current density of the superconducting material, $d$ is the sample diameter, and $k$ is the geometric factor to account for the finite thickness of the disk bulk. Hence, based on equation (1), a simple way to realize higher $B_{\mathrm{T}}$ is to improve $J_{\mathrm{c}}$ by improving its superconducting material properties [27] or to make its diameter larger [28].

Superconducting bulks, magnetized by zero-field-cooled magnetization (ZFCM), can expel the external magnetic flux from its inside due to its "diamagnetic effect" and, as shown for the magnetic lens in the HTFML, can enhance a magnetic field in the central bore of the lens. Several studies have investigated the electromagnetic properties and thermal stability of such magnetic lenses from the viewpoints of superconducting material selection, geometry and mechanical reinforcement [29-31]. A concentrated field of $13 \mathrm{~T}$ at $20 \mathrm{~K}$ was achieved under a background field of $7 \mathrm{~T}$, in which a $\mathrm{GdBaCuO}$ lens was impregnated with epoxy resin to prevent mechanical fracture during its magnetization [31].

The authors proposed a hybrid trapped field magnet lens (HTFML) consisting of two superconducting bulk components - an outer TFM cylinder and an inner bulk magnetic lens - in 2018 [32]. The HTFML can quasi-permanently generate a concentrated magnetic field, $B_{\mathrm{c}}$, higher than the applied field, $B_{\text {app }}$, from an external magnetizing coil, even after this external field is removed, exploiting the combination of the "vortex pinning effect" of the outer TFM and the "diamagnetic lens effect" of the inner magnetic lens. In this conceptual paper, $B_{\mathrm{c}}=13.49 \mathrm{~T}$ for $B_{\mathrm{app}}=10 \mathrm{~T}$ was predicted numerically for a $\mathrm{GdBaCuO} \mathrm{TFM}$ cylinder and $\mathrm{GdBaCuO}$ lens pair [32]. The device was verified experimentally, for which a concentrated magnetic field $B_{\mathrm{c}}=3.55 \mathrm{~T}$ was achieved in the central bore of the HTFML using an $\mathrm{MgB}_{2}$ TFM cylinder and $\mathrm{GdBaCuO}$ magnetic lens, magnetized with an applied field of $B_{\text {app }}=2.0 \mathrm{~T}$ [33]. These studies showed that the trapped field of the bulk could be enhanced by appropriate selection of the bulk materials for the TFM cylinder and lens, as well as the bulk $J_{\mathrm{c}}$ and diameter, following equation (1). In addition to this function, it is considered that the HTFML could generate an ultra-high magnetic field gradient product, $B_{\mathrm{Z}} \cdot d B_{\mathrm{Z}} / d z$, which could, for example, be applicable to magnetic separation.

In this study, three-dimensional numerical models of the HTFML are constructed to investigate the magnetic field performance, in which realistic sizes and shapes of the bulk components and realistic $J_{\mathrm{c}}$ characteristics are assumed, based on an experimental FCM setup. It is predicted that the HTFML could be highly applicable to magnetic levitation as a compact and strong magnetic field source and can generate a magnetic field gradient product, $B_{\mathrm{z}} \cdot d B_{\mathrm{z}} / d z$, over $-3,000 \mathrm{~T}^{2} / \mathrm{m}$, which is six times higher 
than that of a conventional $10 \mathrm{~T}$ superconducting magnet $\left(-500 \mathrm{~T}^{2} / \mathrm{m}\right)$. These results provide further new understanding of the HTFML device and its potential wider use in industrial applications as a strong magnetic field source.

\section{Numerical simulation framework}

Commercial software, Photo-EDDY (Photon Ltd, Japan), was used to analyze the magnetic field profile during the magnetization process, combined with Photo-THERMO to maintain a constant temperature for each bulk component. Electromagnetic phenomena during the magnetization process are described by the fundamental equations introduced in detail in [34-36]. The $E-J$ power law is assumed to represent the highly nonlinear electrical properties of the superconducting material as follows;

$$
E=E_{c}\left(\frac{J}{J_{c}}\right)^{n}
$$

where $E_{\mathrm{c}}\left(=10^{-4} \mathrm{~V} / \mathrm{m}\right)$ is the characteristic electric field, $n=20$ is the power-law exponent for $\mathrm{GdBaCuO}$ and $J_{\mathrm{c}}$ is the critical current density.

Figure 1 shows a schematic view of the three-dimensional numerical model of the HTFML device, consisting of the outer $\mathrm{GdBaCuO}$ TFM cylinder and inner $\mathrm{GdBaCuO}$ lens pair, and the assumed dimensions are exactly the same as those used in the experimental verification of the HTFML presented in [33]. When this HTFML consists of the same superconducting material, e.g. GdBaCuO, for each bulk component, the temperature of each part must be controlled independently, for which a so-called "thermal switch" would be required to control the superconducting state or the normal state during the magnetization process, where both ZFCM process for the inner magnetic lens and FCM process for the outer TFM cylinder are used simultaneously. This magnetizing process could be realized by utilizing an individually controlled cryostat or the same cryostat with different cooling loops for each component. The thermal switch used could be possibly realized mechanically [37], thermally [38] or magnetically.

The $J_{\mathrm{c}}(B)$ characteristics of the $\mathrm{GdBaCuO}$ bulk are described by the following equation, proposed by Jirsa et al. [39],

$$
J_{c}(B)=\beta \times\left\{J_{c 1} \exp \left(-\frac{B}{B_{L}}\right)+J_{c 2} \frac{B}{B_{\max }} \exp \left[\frac{1}{\alpha}\left(1-\left(\frac{B}{B_{\max }}\right)^{\alpha}\right)\right]\right\}
$$

where each relevant parameter, $J_{\mathrm{c} 1}, B_{\mathrm{L}}, J_{\mathrm{c} 2}, B_{\max }$, and $\alpha$ are fitted from the experimental $J_{\mathrm{c}}(B)$ profile. In this work, the experimentally measured $J_{\mathrm{c}}(B)$ data for bulk $\mathrm{GdBaCuO}$ reported by Kii et al. in [40] is assumed, which come from a small sample. The fitting coefficient, $\beta$, was also employed to reproduce the actual trapped field from experimental results.

To obtain the $\beta$ value at each temperature, we refer to our experimental FCM result for the $\mathrm{GdBaCuO}$ bulk ring. Figure 2 presents the temperature dependence of the trapped field, $B_{\mathrm{T}}$, in the 
ring-shaped $\mathrm{GdBaCuO}$ bulk ( $40 \mathrm{~mm}$ in inner diameter, $64 \mathrm{~mm}$ in outer diameter and $20 \mathrm{~mm}$ in height), which was magnetized by FCM from an applied field of $10 \mathrm{~T}$ at $50 \mathrm{~K}$ and then measured in the heating run as the temperature is increased to $93 \mathrm{~K}$, i.e., above its $T_{\mathrm{c}}$ [41]. The experimental $B_{\mathrm{T}}(T)$ curve would have a tendency to saturate under $50 \mathrm{~K}$ due to the full magnetization of the bulk within its $J_{\mathrm{c}}$ capability. When the $J_{\mathrm{c}}(B)$ characteristics obtained by SQUID magnetometer for a small piece of bulk sample $(\beta=1)$ are used to estimate the $B_{\mathrm{T}}$ value numerically at low temperature, e.g., at $40 \mathrm{~K}$ and $20 \mathrm{~K}$, the trapped field was overestimated and this saturation tendency cannot be reproduced. To reproduce the saturation tendency of $B_{\mathrm{T}}(T)$ numerically, $\beta$ should be adjusted to be 0.50 for $40 \mathrm{~K}$ and 0.29 for $20 \mathrm{~K}$, based on the extrapolation of the experimental $B_{\mathrm{T}}(T)$ curve at lower temperatures. The parameters related to the data fitting are summarized in Table 1.

Figure 3 shows the time step sequence of the temperature of the TFM cylinder, $T_{\mathrm{TFM}}$, and the magnetic lens, $T_{\text {lens, }}$ and the external magnetic field, $B_{\mathrm{ex}}$, at the center of the magnetic lens during the magnetizing process. At step 0, before the external field is applied, the bulk cylinder is cooled down to $T_{\mathrm{H}}$, e.g., $100 \mathrm{~K}$, above its $T_{\mathrm{c}}(=92 \mathrm{~K})$ from room temperature, and the bulk lens is cooled to $T_{\mathrm{L}}(<$ $T_{\mathrm{H}}$ ). In this study, two cases $-T_{\mathrm{L}}=40 \mathrm{~K}$ and $T_{\mathrm{L}}=20 \mathrm{~K}-$ are investigated for comparison. The bulk temperature is assumed to be constant, under isothermal conditions; thus, any heat generation is ignored during the whole magnetizing process, assuming a near-static magnetic field. The magnetizing field, $B_{\mathrm{app}}$, which corresponds to the maximum values of $B_{\mathrm{ex}}=3,5$ and $10 \mathrm{~T}$, is applied using a solenoid coil. The magnetizing procedure is divided into two stages: (1) the ascending stage from step 0 to step 5, and (2) the descending stage from step 5 to step 10, where the externally applied field is ramped up and down linearly at $\pm 0.222 \mathrm{~T} / \mathrm{min}$.. 


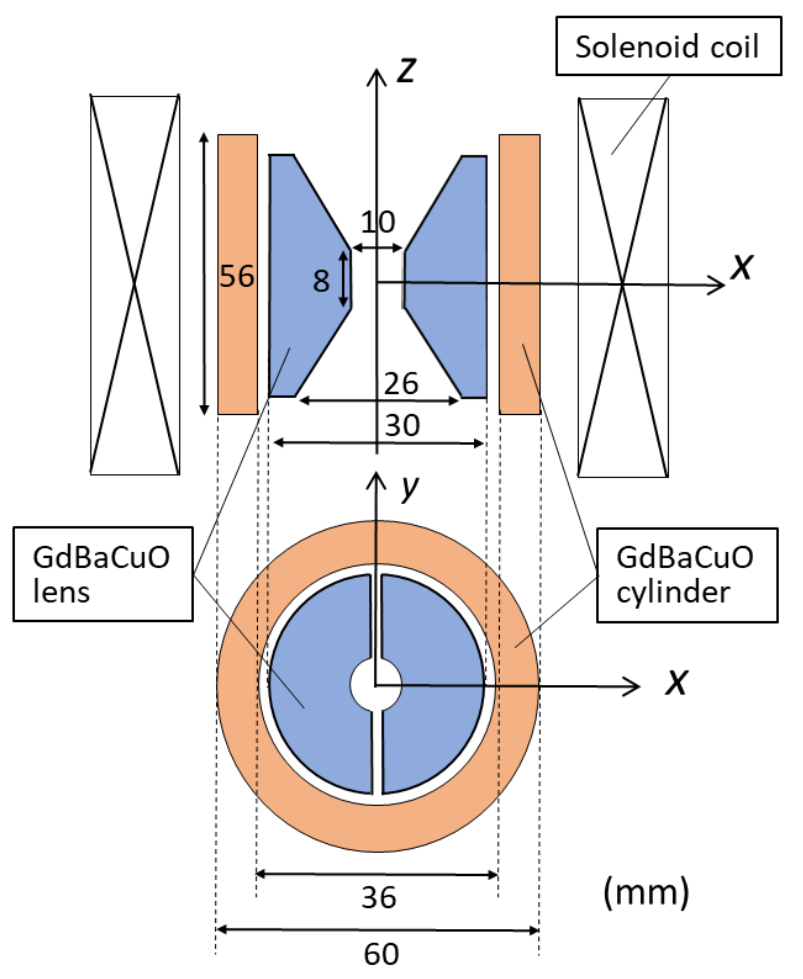

Figure 1. Schematic view of the three-dimensional numerical model of the HTFML, consisting of the outer $\mathrm{GdBaCuO}$ TFM cylinder and inner $\mathrm{GdBaCuO}$ lens pair. The assumed dimensions are exactly the same as those used in the experimental verification of the HTFML [33], reproduced with permission from S Namba et al., Supercond. Sci. Technol. 32 12LT03 (2019). Copyright 2019 IOP Publishing Ltd.

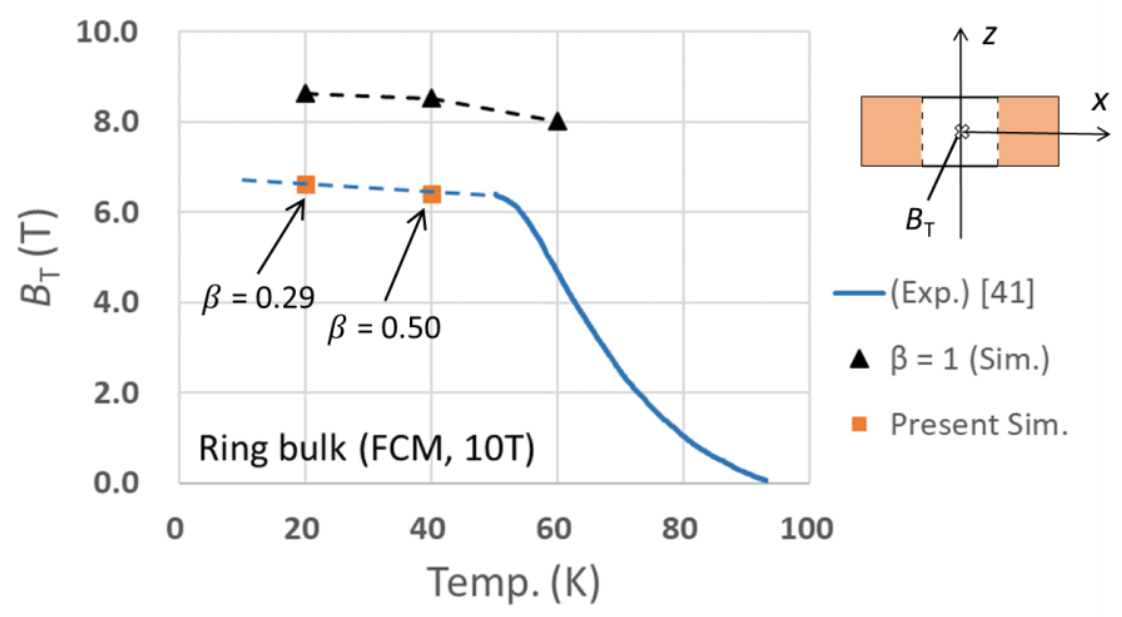

Figure 2. The temperature dependence of the trapped field, $B_{\mathrm{T}}$, in the central bore of the ring-shaped $\mathrm{GdBaCuO}$ bulk ( $40 \mathrm{~mm}$ in inner diameter, $64 \mathrm{~mm}$ in outer diameter and $20 \mathrm{~mm}$ in height; referred to as TFM cylinder in Fig. 1), which was magnetized by FCM from an applied field of $10 \mathrm{~T}$ at $50 \mathrm{~K}$ and then measured in the heating run as the temperature is increased to $93 \mathrm{~K}$. Numerical results of $B_{\mathrm{T}}$ by FCM from $10 \mathrm{~T}$ for $\beta=1$ shown in eq. (3) and the most suitable $B_{\mathrm{T}}$ estimations at $20 \mathrm{~K}$ and $40 \mathrm{~K}$ are also shown (see text). Experimental curve is reproduced with permission from H Fujishiro et al., Supercond. Sci. Technol. 32065001 (2019). Copyright 2019 IOP Publishing Ltd. 
Table 1. Numerical fitting parameters for the $J_{\mathrm{c}}(B)$ characteristics of bulk $\mathrm{GdBaCuO}$ used in eq. (3), for $20 \mathrm{~K}$ and $40 \mathrm{~K}$, where the $\beta$ value is adjusted to reproduce the experimental result for the trapped field in Fig. 2 (see text).

\begin{tabular}{ccccccc}
\hline$T(\mathrm{~K})$ & $J_{\mathrm{c} 1}\left(\mathrm{~A} / \mathrm{m}^{2}\right)$ & $B_{\mathrm{L}}(\mathrm{T})$ & $J_{\mathrm{c} 2}\left(\mathrm{~A} / \mathrm{m}^{2}\right)$ & $B_{\max }(\mathrm{T})$ & $\alpha$ & $\beta$ \\
\hline 20 & $9.0 \times 10^{9}$ & 1.5 & $5.4 \times 10^{9}$ & 8.0 & 0.5 & 0.29 \\
\hline 40 & $3.5 \times 10^{9}$ & 0.9 & $2.7 \times 10^{9}$ & 6.0 & 0.8 & 0.50 \\
\hline
\end{tabular}

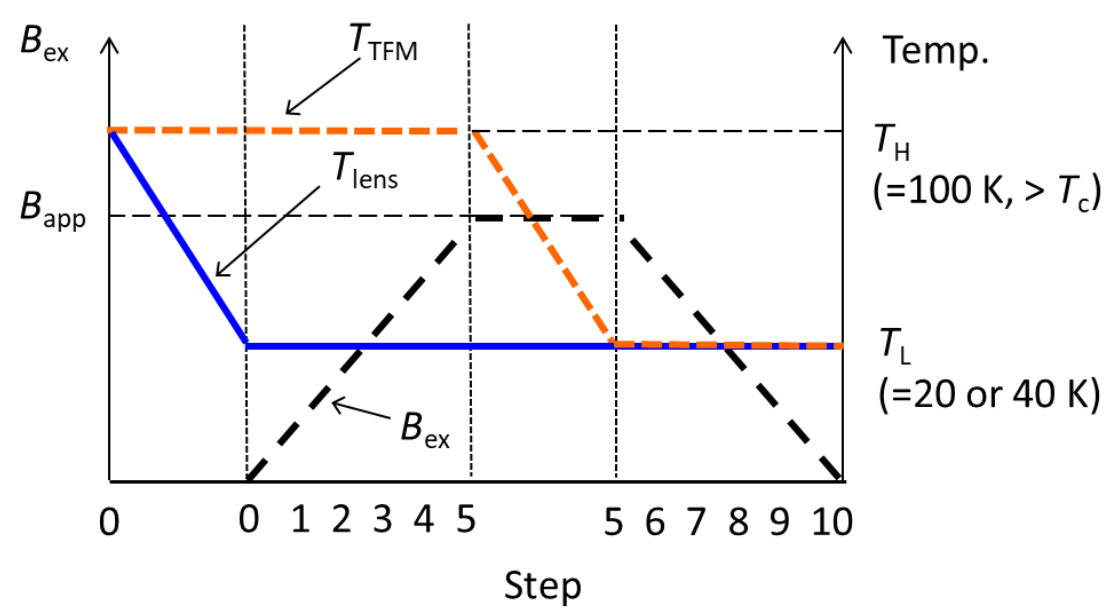

Figure 3. Time step sequence of the temperature of the TFM cylinder, $T_{\mathrm{TFM}}$, and magnetic lens, $T_{\text {lens, }}$, and the external magnetic field, $B_{\mathrm{ex}}$, at the center of the magnetic lens during the magnetizing process, including the ascending stage from step 0 to 5 , and the descending stage until step 10.

\section{Numerical simulation results}

The concentrated magnetic field, $B_{\mathrm{c}}$, in the HTFML changes depending on the maximum applied field, $B_{\text {app }}$, and magnetizing temperature, $T_{\mathrm{L}}$, which can also be deduced from the $J_{\mathrm{c}}(B, T)$ characteristics indicated by equation (1). In this section, $B_{\text {app }}=3,5$ and $10 \mathrm{~T}$ was applied at $T_{\mathrm{L}}=20$ $\mathrm{K}$ and $40 \mathrm{~K}$ in an identical HTFML device using all $\mathrm{GdBaCuO}$ bulk materials, and those results are used to assess the performance of the HTFML in terms of magnetic field and magnetic field gradient product, $B_{\mathrm{z}} \cdot d B_{\mathrm{Z}} / d z$.

Firstly, the magnetic flux dynamics and magnetization mechanisms of the HTFML are explained, by comparing the magnetic field profiles during the magnetization process for each externally applied field. Figure 4 shows the magnetic field profiles along the $x$ (radial)-direction at the middle of the magnetic lens, $z=0 \mathrm{~mm}$, during the magnetization process for the applied fields of $B_{\text {app }}=3,5$ and 10 $\mathrm{T}$ for $T_{\mathrm{L}}=20 \mathrm{~K}$, which is divided into (a) the ascending stage from steps 0 to 5 , and (b) the descending stage from steps 5 to 10 . In the ascending stage, when the TFM cylinder is kept in the normal state at $100 \mathrm{~K}$, but the magnetic lens is already in the superconducting state at $20 \mathrm{~K}$, the diamagnetic effect 
of the magnetic lens expels the external field from its inside within its $J_{\mathrm{c}}(B)$ capability. As a result, a concentrated magnetic field of $B_{\mathrm{c}}=4.9 \mathrm{~T}$ was obtained in the bore of the lens for $B_{\text {app }}=3 \mathrm{~T}$ at step 5, which becomes higher with increasing $B_{\text {app }}$; i.e., $B_{\mathrm{c}}=7.4 \mathrm{~T}$ for $B_{\text {app }}=5 \mathrm{~T}$ and $B_{\mathrm{c}}=12.5 \mathrm{~T}$ for $B_{\text {app }}=$ $10 \mathrm{~T}$. It is worth mentioning here that some magnetic flux penetrates into the magnetic lens, positioned from $x=5$ to $15 \mathrm{~mm}$, which becomes more obvious for higher $B_{\text {app }}$, and this will degrade the resultant concentrated field of the HTFML. In the descending stage, which includes the FCM process for the TFM cylinder at $20 \mathrm{~K}$, the magnetic flux is gradually trapped in the TFM cylinder positioned at $x=$ 18 to $30 \mathrm{~mm}$ with increasing time step after step 5. The trapped field in the TFM cylinder at step 10 is roughly equal to $B_{\text {app }}$ (or slightly higher because the applied field profile is not completely homogeneous), which corresponds to the so-called partial magnetization of the TFM cylinder below its full capability (see Fig. 5(b), for example). The concentrated magnetic field in the magnetic lens appears to degrade little during the descending stage, compared to the ascending stage, indicating that the ascending stage of the magnetization would determine the remnant concentrated field in the HTFML after its magnetization. These numerical results during the magnetizing process are helpful towards achieving a higher magnetic field and a higher $B_{\mathrm{z}} \cdot d B_{\mathrm{Z}} / d z$ value in the HTFML to improve its performance.

Figures 5(a) and (b) show the cross-sectional profiles of the magnetic field, $B_{\mathrm{z}}$, along the $z$ (height)direction and the induced current density, $J_{\mathrm{y}}$, along the $y$ (circumferential)-direction in the HTFML, after the magnetization process at step 10 for each $B_{\text {app }}$ at $T_{\mathrm{L}}=20 \mathrm{~K}$. As already shown in Fig. 4(a), the flux penetration into the magnetic lens is more remarkable for higher applied fields, as shown in Fig. 5(a). In the case of $B_{\text {app }}=10 \mathrm{~T}$, there is no region of $0 \mathrm{~T}$ inside the magnetic lens, meaning that the magnetic flux completely penetrates the inside of the lens during the magnetization process. In Fig. 5(b), inside each part of the magnetic lens, the induced current flows in the opposite direction to that in the TFM cylinder due to the existence of the slits in the hollow cone (see Fig. 1). The $J_{\mathrm{y}}$ profile

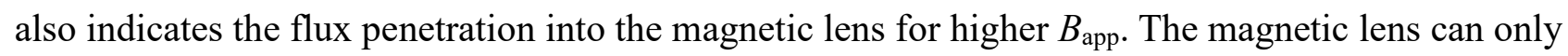
exhibit the diamagnetic effect with an induced current flowing predominately on its surface, whereas the TFM cylinder generates its strongest trapped field by an induced current flowing through the entire cross-section of the TFM cylinder.

Figures 6(a) and 6(b) show the time step dependence of the concentrated magnetic field, $B_{\mathrm{c}}$, in the central bore of the magnetic lens $(x=y=z=0 \mathrm{~mm})$ and the magnetic field concentration ratio, $B_{\mathrm{c}} / B_{\text {app}}$, respectively, extracted from fig. 4 for each $B_{\text {app }}$ at $T_{\mathrm{L}}=20 \mathrm{~K}$. The concentration ratio can be referred to characterize the diamagnetic lens effect, which is determined by the shape of lens, including the outer and inner diameter, the outer and inner height and the angle of the slits [42]. From these results, a higher applied field is needed to achieve a higher concentrated field at any time step, as shown in Fig. 6 (a), although the concentration ratio gradually degrades as the magnetization process proceeds from step 0 to step 10, as shown in Fig. 6(b). The so-called decay behavior in the bulk magnet due to 
flux creep usually exhibits an approximately logarithmic time dependence, as reported in the case only using the magnetic lens. If the outer TFM cylinder can provide a homogeneous magnetic field and replace outer magnetizing coil used for magnetizing process, the similar trend would be obtained also in this HTFML as well, which means a concentrated field of $5 \mathrm{~T}$ could be maintained by only about $0.1 \%$ drop per hour after magnetization; but that decay rate depends on operating temperature and applied magnetic field [31]. It may be needed to develop a new magnetizing sequence to improve a temporal stabilization of magnetic flux after magnetizing process, specifically for magnetic separation.

Figures 7(a) and 7(b) show the resultant magnetic field, $B_{\mathrm{z}}$, profiles $(x=y=0 \mathrm{~mm})$ along the $z$ (height) direction, after magnetization from $B_{\text {app }}=10 \mathrm{~T}$ at $T_{\mathrm{L}}=20 \mathrm{~K}$, and the magnetic field gradient product, $B_{\mathrm{Z}} \cdot d B_{\mathrm{Z}} / d z$, profile ( $x=y=0 \mathrm{~mm}$ ), respectively. A schematic image of the HTFML is also shown for reference, indicating the corresponding position in the HTFML. A characteristic feature of the HTMFL is based on the existence of magnetic lens located inside the homogeneous trapped field generated from the TFM cylinder, which enables the generation of a higher inhomogeneous magnetic field by exploiting the diamagnetic effect of magnetic lens. As a result, the HTFML also has a significant ability to produce an intentional ultra-high magnetic field gradient product of $B_{\mathrm{z}} \cdot d B_{\mathrm{Z}} / d z$ $\pm 3,000 \mathrm{~T}^{2} / \mathrm{m}$, just below and above the lens center at $z= \pm 5 \mathrm{~mm}$, for a concentrated field of $B_{\mathrm{c}}=11$. $4 \mathrm{~T}$ for $B_{\text {app }}=10 \mathrm{~T}$. This value is comparable with that produced by a $>20 \mathrm{~T}$-class, large-scale hybrid magnet [43].

Another parameter that determines the HTFML performance is the magnetizing temperature $T_{\mathrm{L}}$ in Fig. 3. Hence, the HTFML performance was explored for the higher magnetizing temperature $T_{\mathrm{L}}=$ $40 \mathrm{~K}$ to compare with $T_{\mathrm{L}}=20 \mathrm{~K}$. Figure 8 summarizes the applied field dependence of the HTFML properties, including (a) the concentrated magnetic field, $B_{\mathrm{c}}$, at the center of the $\mathrm{GdBaCuO}$ lens, (b) maximum $\left|B_{z} \cdot d B_{Z} / d z\right|$ and (c) concentration ratio, $B_{\mathrm{c}} / B_{\text {app }}$, for each $T_{\mathrm{L}}$. The difference in $B_{\mathrm{c}}$ is reasonably small for each $T_{\mathrm{L}}$ in Fig. $8(\mathrm{a})$, but $\left|B_{z} \cdot d B_{z} / d z\right|$ in Fig. $8(\mathrm{~b})$ at $T_{\mathrm{L}}=20 \mathrm{~K}$ is $500 \mathrm{~T}^{2} / \mathrm{m}$ larger than that at $T_{\mathrm{L}}=40 \mathrm{~K}$ for the applied field $B_{\text {app }}=10 \mathrm{~T}$. A lower $T_{\mathrm{L}}$ is desirable for a higher applied field, resulting in a higher $B_{\mathrm{c}}$ and higher $\left|B_{z} \cdot d B_{z} / d z\right|$, although the diamagnetic effect degrades with increasing $B_{\text {app }}$ for each $T_{\mathrm{L}}$, as shown by $B_{\mathrm{c}} / B_{\text {app }}$ in Fig. 8(c). The obtained $B_{\mathrm{c}}$ and the maximum $B_{\mathrm{z}} \cdot d B_{\mathrm{z}} / d z$ are summarized for each applied field, $B_{\mathrm{app}}$, in Table 2 . There is scope for further improvement of the HTFML performance as a strong magnet in terms of both the magnetic design of each bulk component and their magnetizing conditions. 

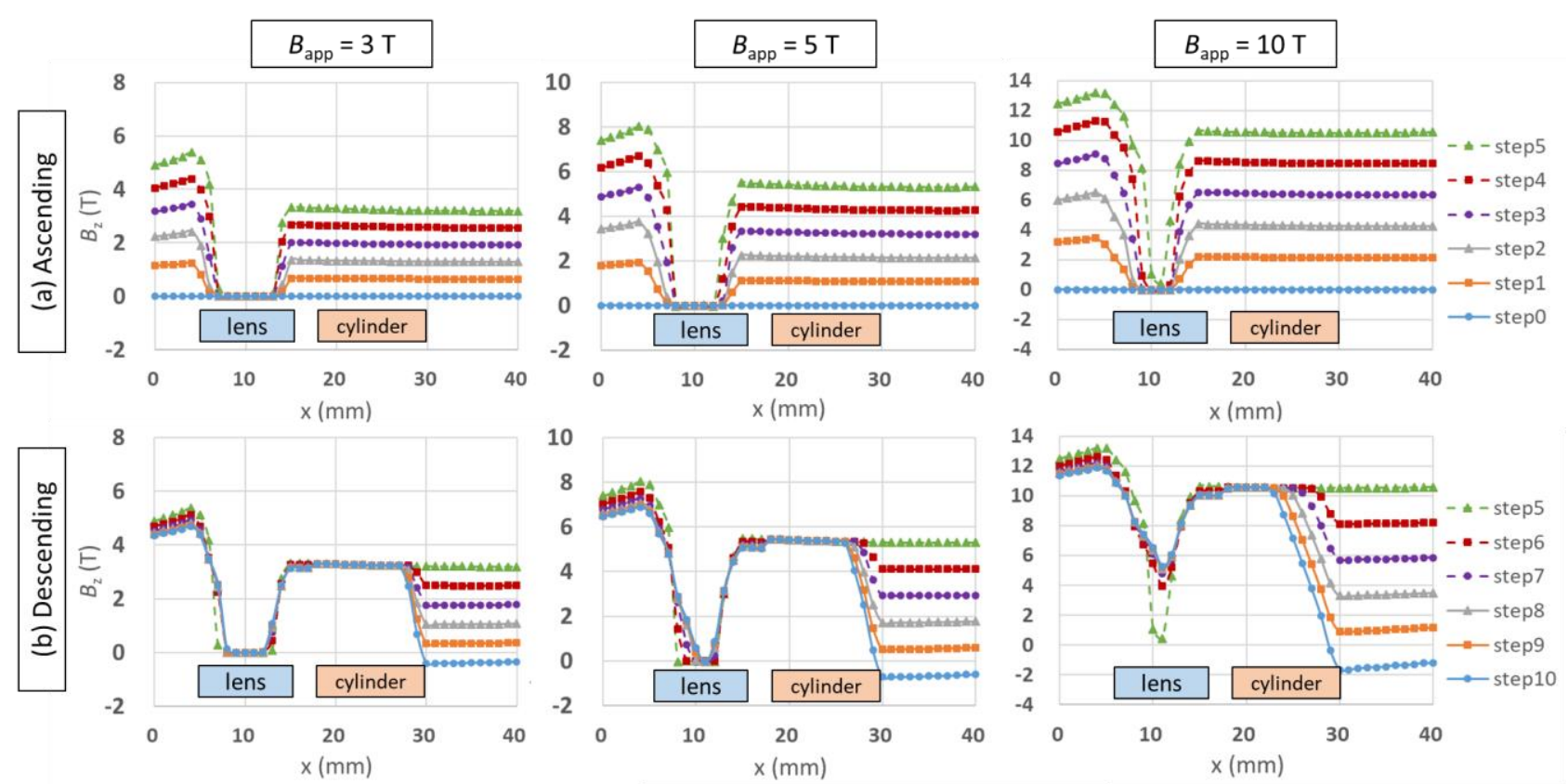

Figure 4. Magnetic field profiles along the $x$ (radial)-direction at the middle of the magnetic lens, $z=$ $0 \mathrm{~mm}$, during the magnetization process from an applied field $B_{\text {app }}=3,5$ and $10 \mathrm{~T}$ at $T_{\mathrm{L}}=20 \mathrm{~K}$, which are divided into (a) the ascending stage from steps 0 to 5 and (b) the descending stage from steps 5 to 10 .

(a)

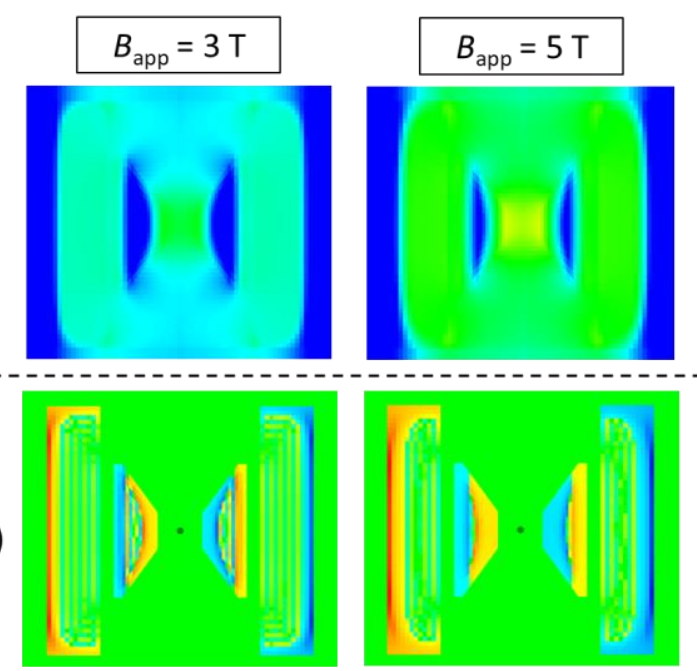

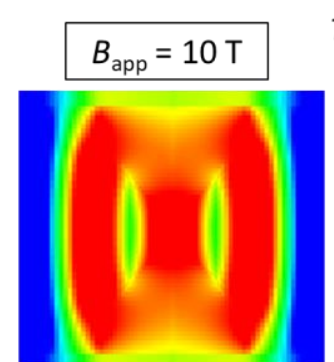

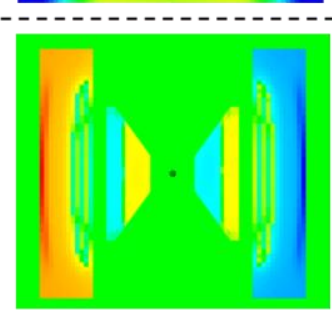

$$
T_{\mathrm{L}}=20 \mathrm{~K}
$$
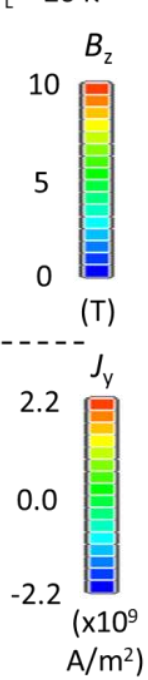
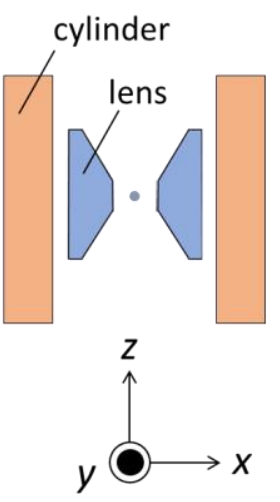

Figure 5. Cross-section of (a) the magnetic field, $B_{z}$, profile along the $z$ (height)-direction and (b) the induced current density, $J_{\mathrm{y}}$, along the $y$ (circumferential)-direction in the HTFML, after the magnetization process at step 10 for each applied field, $B_{\text {app }}$, at $T_{\mathrm{L}}=20 \mathrm{~K}$. 


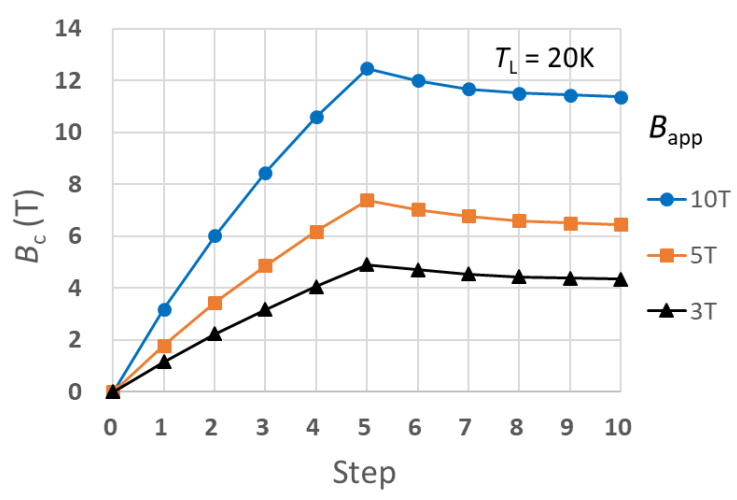

(a)

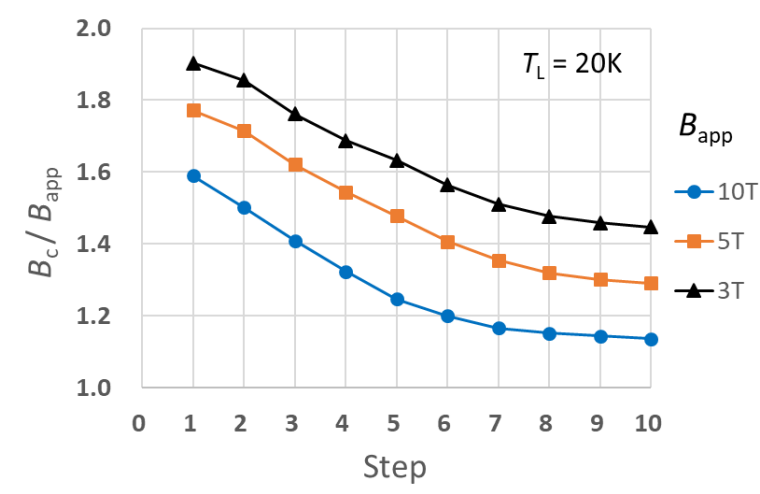

(b)

Figure 6. Time step dependence of (a) the concentrated magnetic field, $B_{\mathrm{c}}$, in the central bore of $\mathrm{GdBaCuO}$ lens $(x=y=z=0 \mathrm{~mm})$, and (b) the magnetic field concentration ratio, $B_{\mathrm{c}} / B_{\mathrm{app}}$, extracted from Fig. 4 for each $B_{\text {app }}$ at $T_{\mathrm{L}}=20 \mathrm{~K}$.

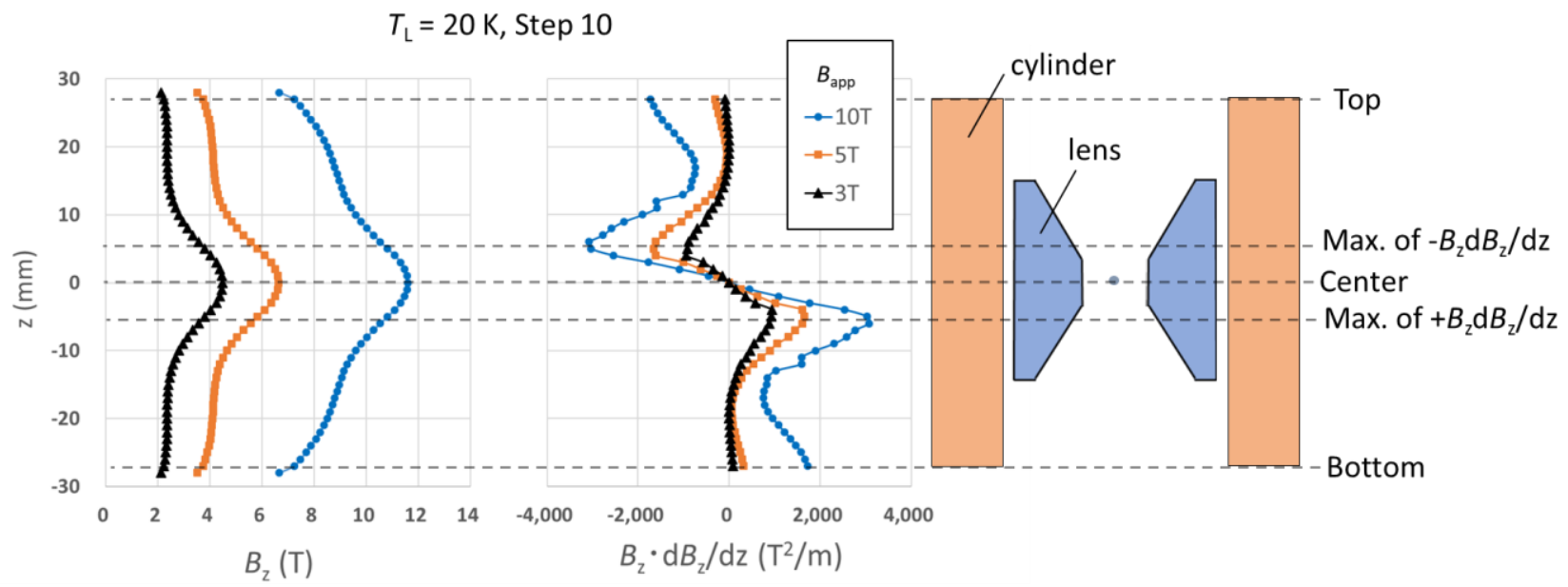

(a)

(b)

Figure 7. Resultant magnetic field, $B_{\mathrm{z}}$, profiles $(x=y=0 \mathrm{~mm})$ along the $z$ (height)-direction after the magnetization process (step 10) from $B_{\text {app }}=10 \mathrm{~T}$ at $T_{\mathrm{L}}=20 \mathrm{~K}$, and the magnetic field gradient product, $B_{\mathrm{z}} \cdot d B_{\mathrm{z}} / d z$, profiles $(x=y=0 \mathrm{~mm})$. A schematic image of the HTFML is also shown for reference, indicating the corresponding position in the HTFML. 

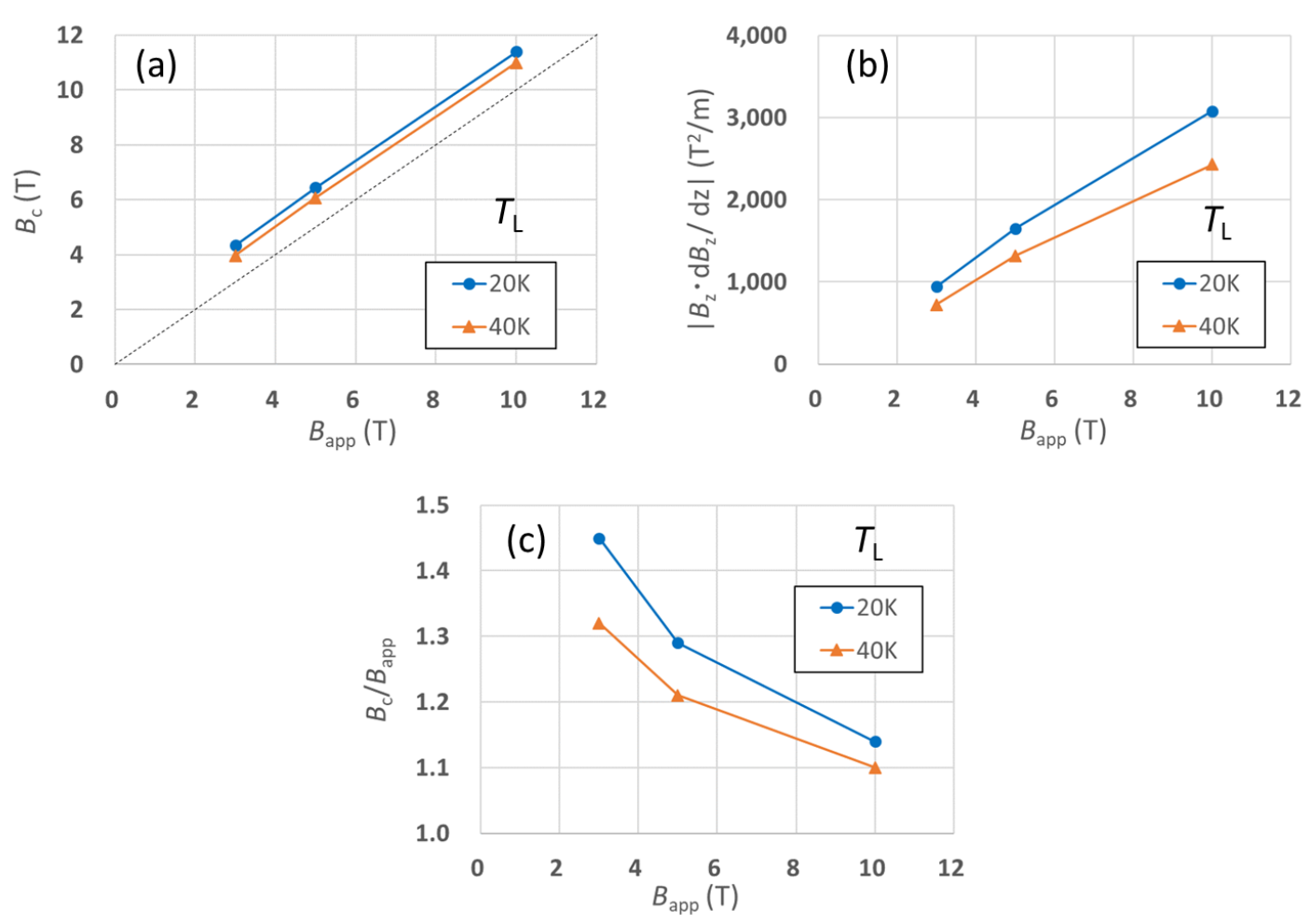

Figure 8. Applied field dependence of the HTFML properties for (a) the concentrated magnetic field, $B_{\mathrm{c}}$, at the center of the $\mathrm{GdBaCuO}$ lens, (b) the maximum of the magnetic field gradient product, $\left|B_{z} \cdot d B_{z} / d z\right|$, and (c) the concentration ratio, $B_{\mathrm{c}} / B_{\text {app }}$, for each $T_{\mathrm{L}}$.

Table 2. Maximum applied field, $B_{\text {app }}$, concentrated magnetic field, $B_{\mathrm{c}}$, at the center of the $\mathrm{GdBaCuO}$ lens at the final step of the magnetizing process (step 10), magnetic field concentration ratio, $B_{\mathrm{c}} / B_{\mathrm{app}}$, and maximum of the magnetic field gradient product, $B_{\mathrm{Z}} \cdot d B_{\mathrm{Z}} / d z$, at $T_{\mathrm{L}}=20 \mathrm{~K}$ and $40 \mathrm{~K}$.

\begin{tabular}{ccccc}
$T_{\mathrm{L}}(\mathrm{K})$ & $B_{\text {app }}(\mathrm{T})$ & $B_{\mathrm{c}}(\mathrm{T})$ & $B_{\mathrm{c}} / B_{\text {app }}$ & $B_{\mathrm{z}} \cdot \mathrm{d} B_{\mathrm{Z}} / \mathrm{dz}\left(\mathrm{T}^{2} / \mathrm{m}\right)$ \\
\hline \multirow{2}{*}{$20 \mathrm{~K}$} & 10 & 11.4 & 1.14 & $-3,080$ \\
\cline { 2 - 5 } & 5 & 6.45 & 1.29 & $-1,650$ \\
\hline & 3 & 4.34 & 1.45 & -943 \\
\hline \multirow{2}{*}{$40 \mathrm{~K}$} & 10 & 11.0 & 1.10 & $-2,430$ \\
\cline { 2 - 5 } & 5 & 6.07 & 1.21 & $-1,320$ \\
\hline
\end{tabular}

\section{$\underline{\text { 4. Possibility of magnetic separation using the ultra-high magnetic field gradient product }}$}

In this section, the superiority of the HTFML as a strong magnet applied to magnetic levitation or separation is discussed. The HTFML could, for example, be highly applicable to magnetic separation, which needs a large magnetic field gradient product, $B_{\mathrm{Z}} \cdot \mathrm{d} B_{\mathrm{Z}} / \mathrm{d} z$, over $-2,000 \mathrm{~T}^{2} / \mathrm{m}$ to realize the so- 
called "apparent zero gravity" condition, even in water or air for any diamagnetic objects with the magnetic susceptibility $\approx-10^{-5}$, such as metals, foods or plastics.

The total potential energy per unit mass of a substance dispersed in a medium, e.g. liquid or gas, is expressed by [44],

$$
U=-\frac{1}{2 \mu_{0}}\left(\chi_{s}-\chi_{m}\right) \boldsymbol{B}^{2}+\left(\rho_{s}-\rho_{m}\right) g z
$$

where $\mu_{0}$ is the vacuum permeability, $\boldsymbol{B}$ is the field intensity, $g$ is the acceleration due to gravity, and $z$ is the vertical position. $\chi_{s}$ and $\rho_{s}$ are the susceptibility and density of the target substance, respectively. $\chi_{m}$ and $\rho_{m}$ are the same parameters for the medium, such as liquid or gas. The total magnetic force, $F_{z}$, along $z$ (central axis)-direction at $x=y=0 \mathrm{~mm}$, is

$$
F_{z}=-\frac{\partial U_{(x=y=0)}}{\partial z}=F_{m}+F_{g}=\frac{\left(\chi_{s}-\chi_{m}\right)}{\mu_{0}} B_{z} \frac{d B_{z}}{d z}-\left(\rho_{s}-\rho_{m}\right) g .
$$

$F_{\mathrm{z}}$ is determined by the $B_{\mathrm{z}} \cdot d B_{\mathrm{z}} / d z$ value, which changes in the vertical bore of the magnet as shown in Fig. 7(b). The equilibrium condition for magneto-Archimedes levitation, which corresponds to $F_{z}$ $=0$, can then be written as

$$
B_{z} \frac{d B_{z}}{d z}=\frac{\rho_{s}-\rho_{m}}{\chi_{s}-\chi_{m}} \mu_{0} g
$$

Figure 9 shows a schematic image of magnetic separation using the HTFML device. Several kinds of substances of different susceptibility and density are dispersed in the medium, e.g., water in the tube. When the tube is inserted into the central bore of the magnetized HTFML device, each substance is re-arranged vertically according to the differences in $\chi_{s}$ and $\rho_{s}$ of each substance. Most simply, in this case, a collecting capillary tube could be used for the recovery process and should be placed above the optional height of each substances floated in the glass tube in the HTFML device.

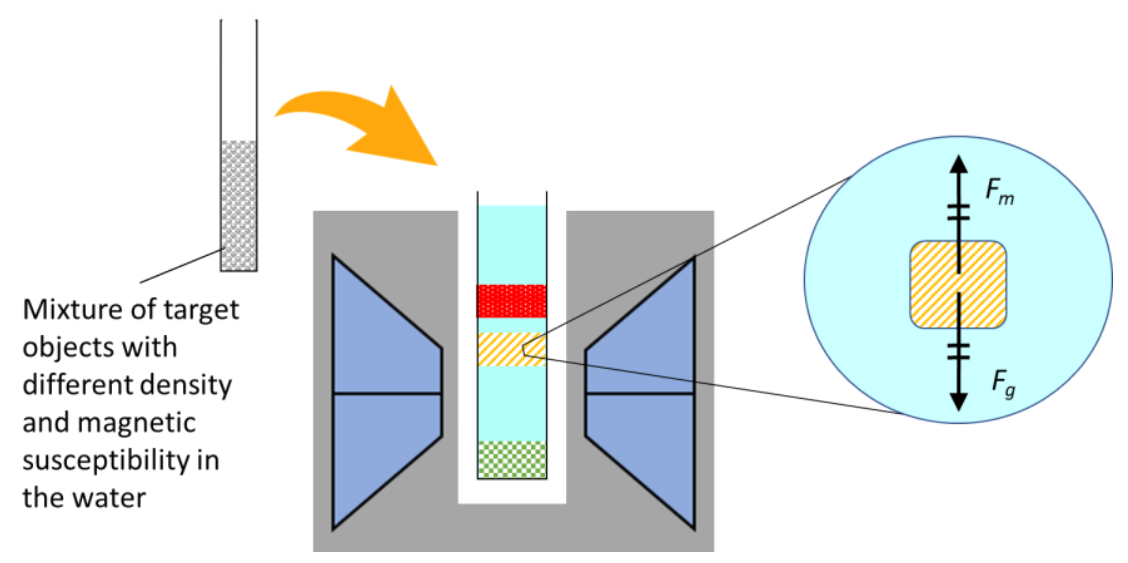

Figure 9. Schematic image of magnetic separation using the magnetized HTFML device, in which the force balance between gravity, $F_{g}$, and the magnetic repulsive force, $F_{m}$, is realized for each substance in the medium, e.g., water, at particular positions related to the magnetic field gradient product. 
Figures 10(a) and 10(b), respectively, show cross-sectional views of $B_{\mathrm{z}}$ and $B_{\mathrm{z}} \cdot d B_{\mathrm{z}} / d z$ in the $x z$ (vertical)-plane at $y=0 \mathrm{~mm}$ and the $x y$ (horizontal)-plane at $z=0 \mathrm{~mm}$ of the HTFML, after magnetization from $B_{\text {app }}=10 \mathrm{~T}$ at $20 \mathrm{~K}$. The effective hollow area for levitation is indicated by the dotted line. As shown in Fig. 10(a), the $B_{\mathrm{Z}} \cdot d B_{\mathrm{Z}} / d z$ value changes from $-3,000$ to $+3,000 \mathrm{~T}^{2} / \mathrm{m}$ along the vertical direction in this area of $\varphi 10 \mathrm{~mm} \mathrm{x} \pm 30 \mathrm{~mm}$ in height in the bore of the magnetic lens for the present HTFML.

For magnetic separation, a large $B_{\mathrm{z}} \cdot d B_{\mathrm{z}} / d z$ would be needed for a variety of cases, especially if the density of the target object is heavy, such as platinum $\left(\rho_{\mathrm{s}}=21.5 \mathrm{~g} / \mathrm{cm}^{3}\right)$, and/or its magnetic susceptibility is exceedingly low, such as water $\left(\chi_{\mathrm{m}}=-9.03 \times 10^{-6}\right)$. The use of harmful paramagnetic medium liquids, such as $\mathrm{MnCl}_{2}$ solution, should be avoided for food and medical use [3].
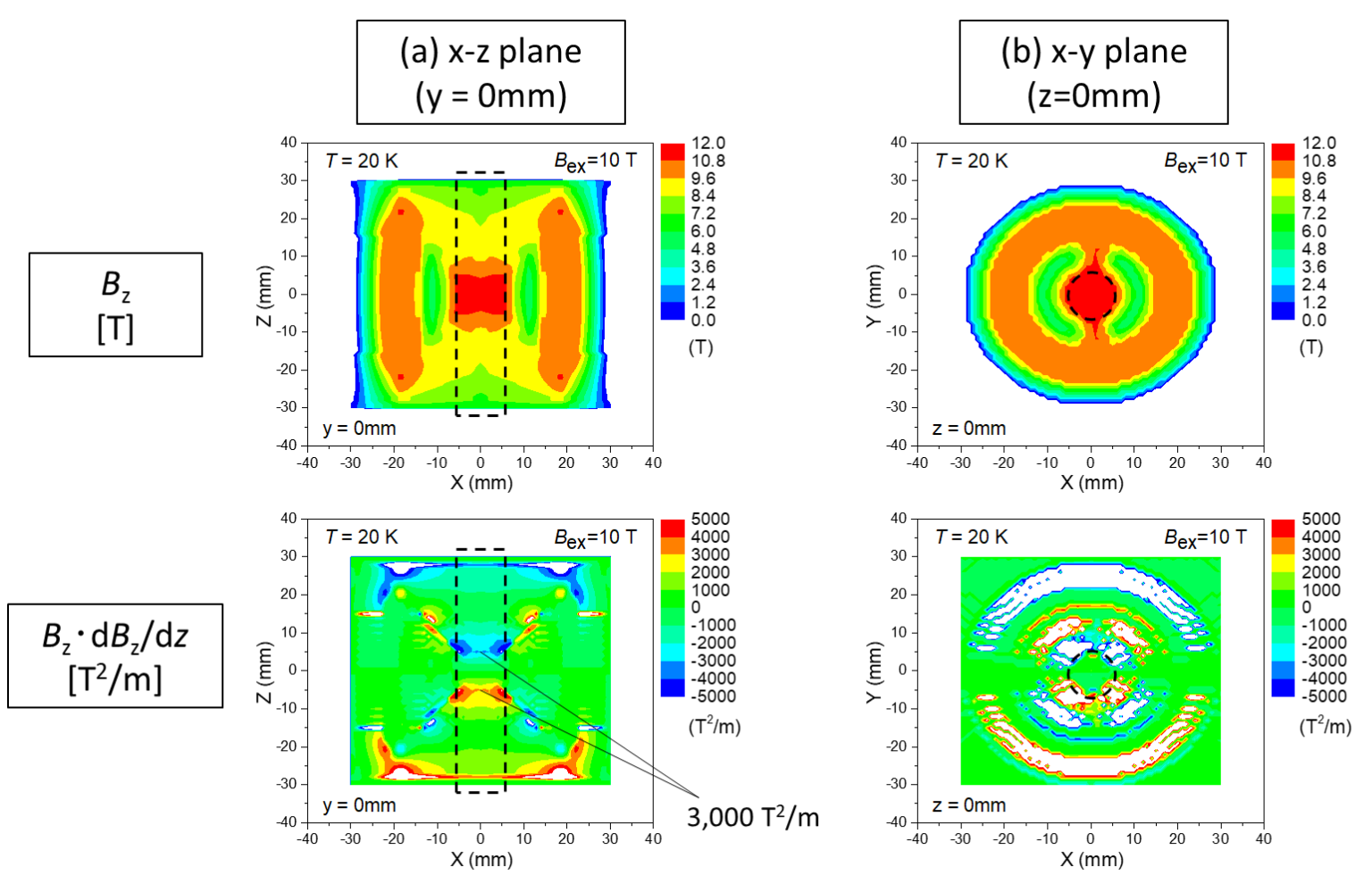

Figure 10. Cross-sectional views of the resultant $B_{\mathrm{z}}$, and $B_{\mathrm{z}} \cdot d B_{\mathrm{z}} / d z$ along (a) the $x z$ (vertical)-plane at $y=0 \mathrm{~mm}$ and (b) the $x y$ (horizontal)-plane at $z=0 \mathrm{~mm}$ of the HTFML, after magnetization from $B_{\text {app }}$ $=10 \mathrm{~T}$ at $20 \mathrm{~K}$.

Hereafter, the levitation position of the target object in the HTFML is estimated for kinds of precious metals (Pt, $\mathrm{Au}, \mathrm{Ag}$ and $\mathrm{Cu}$ ) based on the magneto-Archimedes effect described by equation (4). The relevant physical parameters of the objects, referred from [2], are presented in Table 3. The parameters for aqueous $\mathrm{MnCl}_{2}$ solution with concentrations of $\mathrm{MnCl}_{2}, 50 \mathrm{wt} \%$ and $12 \mathrm{wt} \%$, and 0 $\mathrm{wt} \%$ (i.e., pure water), are also referred from $[2,3,13]$. The required magnetic field gradient product, 
$B_{\mathrm{Z}} \cdot d B_{\mathrm{Z}} / d z$, for levitation increases with decreasing concentration of $\mathrm{MnCl}_{2}$ in the solution. All of the precious metals can levitate in the HTFML for $\mathrm{MnCl}_{2}$ concentrations of $50 \mathrm{wt} \%$ and $12 \mathrm{wt} \%$. However, for the $0 \mathrm{wt} \% \mathrm{MnCl}_{2}$ solution (pure water), only copper needs a higher $B_{\mathrm{z}} \cdot d B_{\mathrm{z}} / d z$ value of over $-7,000 \mathrm{~T}^{2} / \mathrm{m}$ in water due to its high density and low susceptibility, which exceeds the capability of the present HTFML device. As a result, copper sinks in water, but the other precious metals can stably levitate at different heights in the bore of the HTFML.

Figure 11 shows the estimated levitation position of each precious metal in the pure water solution in the central bore of the HTFML magnetized by $B_{\text {app }}=10 \mathrm{~T}$ at $20 \mathrm{~K}$. Magnetic levitation would be realized in the case when the object is stably lifted from beneath by the repulsive magnetic force; that is, the effective area exists above a negative peak of $B_{\mathrm{z}} \cdot d B_{\mathrm{Z}} / d z$ at $z=+5 \mathrm{~mm}$ for a diamagnetic object and above a positive peak at $z=-5 \mathrm{~mm}$ for a paramagnetic object. For the precious metals in Table. 3 , magnetic levitation could be realized at $z=+11 \mathrm{~mm}$ for the diamagnetic gold $\left(B_{\mathrm{z}} \cdot d B_{\mathrm{z}} / d z=-1401\right.$ $\left.\mathrm{T}^{2} / \mathrm{m}\right)$ and at $z=+6 \mathrm{~mm}$ for the diamagnetic silver $\left(B_{\mathrm{z}} \cdot d B_{\mathrm{z}} / d z=-2800 \mathrm{~T}^{2} / \mathrm{m}\right)$ above the negative $B_{\mathrm{z}} \cdot d B_{\mathrm{z}} / d z$ peak. On the other hand, magnetic levitation could be realized at $z=-8 \mathrm{~mm}$ for the paramagnetic platinum $\left(B_{\mathrm{Z}} \cdot d B_{\mathrm{Z}} / d z=+2210 \mathrm{~T}^{2} / \mathrm{m}\right)$ above the positive $B_{\mathrm{Z}} \cdot d B_{\mathrm{Z}} / d z$ peak. However, copper would settle at the bottom of the tube because it is outside of the present HTFML's magnetic separation capability.

With respect to the recovery method, a suitable one has not been proposed yet, and it should be determined on the basis of the separation method and target material(s). A separation and recovery experiment was reported by Ando et al. [44], in which glass particles floated by the magnetoArchimedes method were collected using movable partitions into each compartment in their device.

Table 3. Density and susceptibility of the example metals and calculated $B_{\mathrm{z}} \cdot d B_{\mathrm{z}} / d z$ values to levitate them in aqueous $\mathrm{MnCl}_{2}$ solutions with concentrations $50 \mathrm{wt} \%$ and $12 \mathrm{wt} \% \mathrm{MnCl}_{2}$, and $0 \mathrm{wt} \%$ (i.e., pure water). The parameters for aqueous $\mathrm{MnCl}_{2}$ solutions are also shown for reference from [2, 3, 13], reproduced with permission from: O Miura et al., J. Phys.: Conf. Ser. 1054012086 (2018). Copyright 2018 IOP Publishing Ltd., T Kobayashi et al., J. Phys.: Conf. Ser. 897012018 (2017). Copyright 2017 IOP Publishing Ltd., and Y Ikezoe et al., Nature 393749 (1998). Copyright 1998 Springer Nature.

\begin{tabular}{cccccc}
\hline \multirow{2}{*}{ Metal } & $\begin{array}{c}\text { Density }\left(\rho_{s}\right) \\
\left(\mathrm{g} / \mathrm{m}^{3}\right)\end{array}$ & $\begin{array}{c}\text { Susceptibility } \\
\left(\chi_{s}\right)\end{array}$ & $\begin{array}{c}\mathrm{MnCl}_{2} \\
(50 \mathrm{wt} \%)\end{array}$ & $\begin{array}{c}\mathrm{MnCl}_{2} \\
(12 \mathrm{wt} \%)\end{array}$ & $\begin{array}{c}\mathrm{MnCl}_{2} \\
(0 \mathrm{wt} \% ; \text { pure } \\
\text { water })\end{array}$ \\
\hline Platinum & 21.5 & $1.05 \times 10^{-4}$ & -805 & -2388 & +2210 \\
\hline Gold & 19.3 & $-1.70 \times 10^{-4}$ & -380 & -590 & -1401 \\
\hline Silver & 5.56 & $-2.91 \times 10^{-5}$ & -118 & -230 & -2800 \\
\hline Copper & 8.96 & $-2.25 \times 10^{-5}$ & -216 & -417 & -7282 \\
\hline
\end{tabular}




\begin{tabular}{ccc}
\hline Medium & $\begin{array}{c}\text { Density }\left(\rho_{m}\right) \\
\left(\mathrm{g} / \mathrm{m}^{3}\right)\end{array}$ & $\begin{array}{c}\text { Susceptibility } \\
\left(\chi_{m}\right)\end{array}$ \\
\hline $\mathrm{MnCl}_{2}(50 \mathrm{wt} \%)$ & 1.33 & $4.13 \times 10^{-4}$ \\
\hline $\mathrm{MnCl}_{2}(12 \mathrm{wt} \%)$ & 1.10 & $2.10 \times 10^{-4}$ \\
\hline $\begin{array}{c}\mathrm{MnCl}_{2}(0 \mathrm{wt} \% ; \\
\text { pure water })\end{array}$ & 1.00 & $-9.03 \times 10^{-6}$ \\
\hline
\end{tabular}

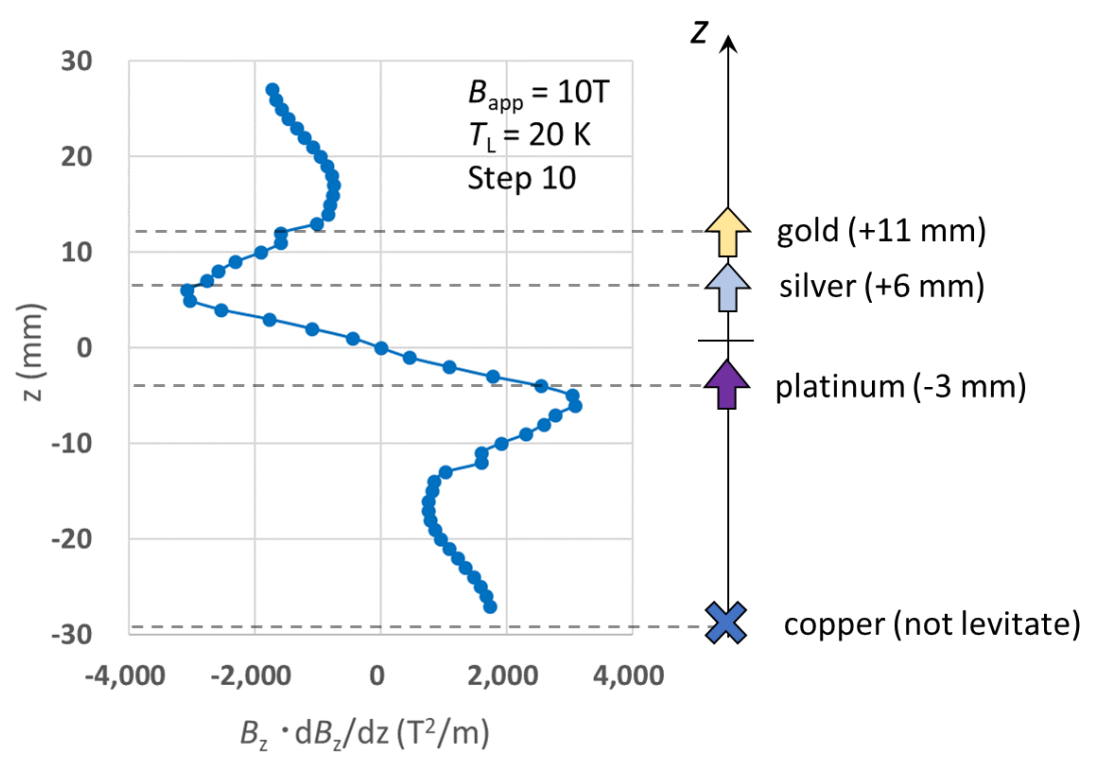

Figure 11. Estimated levitation positions for each precious metal in the pure water solution in the central bore of the HTFML magnetized by $B_{\text {app }}=10 \mathrm{~T}$ at $20 \mathrm{~K}$ (see text).

Thus, the superiority of the HTFML to achieve magnetic separation using its ultra-high magnetic field gradient product has been proposed from the following viewpoints:

(1) Realization of an ultra-high magnetic field gradient product, $B_{\mathrm{z}} \cdot d \underline{B}_{\mathrm{Z}} / d z$ : there are a limited number of facilities worldwide that can generate a $B_{\mathrm{z}} \cdot d B_{\mathrm{z}} / d z \sim 2,000 \mathrm{~T}^{2} / \mathrm{m}$ for magnetic levitation. Figure 12 summarizes the relationship between the applied magnetic field, $B_{\text {app }}$, and the magnetic field gradient product, $B_{\mathrm{z}} \cdot d B_{\mathrm{z}} / d z$, for superconducting magnets (SM) and large-scale hybrid magnets (HM) around the world $[2,11,45-50]$, which are compared to the numerical results for the present HTFML device. The HTFML would generate quasi-permanently, an ultra-high magnetic field gradient product higher than that of conventional SMs and large-scale HMs, even for a lower applied field of $10 \mathrm{~T}$, which is enhanced due to the presence of the magnetic lens.

(2) Compact size and mobility: this kind of high magnetic field source utilizing the HTFML would be a compact and mobile desktop-type superconducting bulk magnet system. Thus, it may be possible to improve the accessibility to strong magnetic fields in a variety of food, medical and industrial applications. 
(3) Mass productivity: Superconducting bulk magnets such as $\mathrm{REBaCuO}$, fabricated by the meltgrowth technique, and $\mathrm{MgB}_{2}$, fabricated by the reactive $\mathrm{Mg}$ liquid infiltration, are available commercially and are being used in commercial products. In addition, a cryogenic refrigerator enables thermal control of the bulks in a compact and light-weight cryostat. The HTFML magnet, which consists of these technology-intensive components, is superior in terms of mass productivity to carry out high-throughput continuous separation in devices with different magnetic field gradient products.

(4) Cost efficiency: Unlike a conventional separation technique based on chemical processes, this HTFML would not need other energy consumptions for magnetic separation due to superconducting current that keeps flowing inside the bulk quasi-permanently, if the operating temperature is preserved, for which the bulk is kept as below $T_{\mathrm{c}}$ in the HTFML. It is possible to establish and operate the HTFML system using a cryogen-free refrigerator, which only needs electric power consumption for operation as a more cost-effective way without any coolants such as liquid nitrogen or liquid helium.

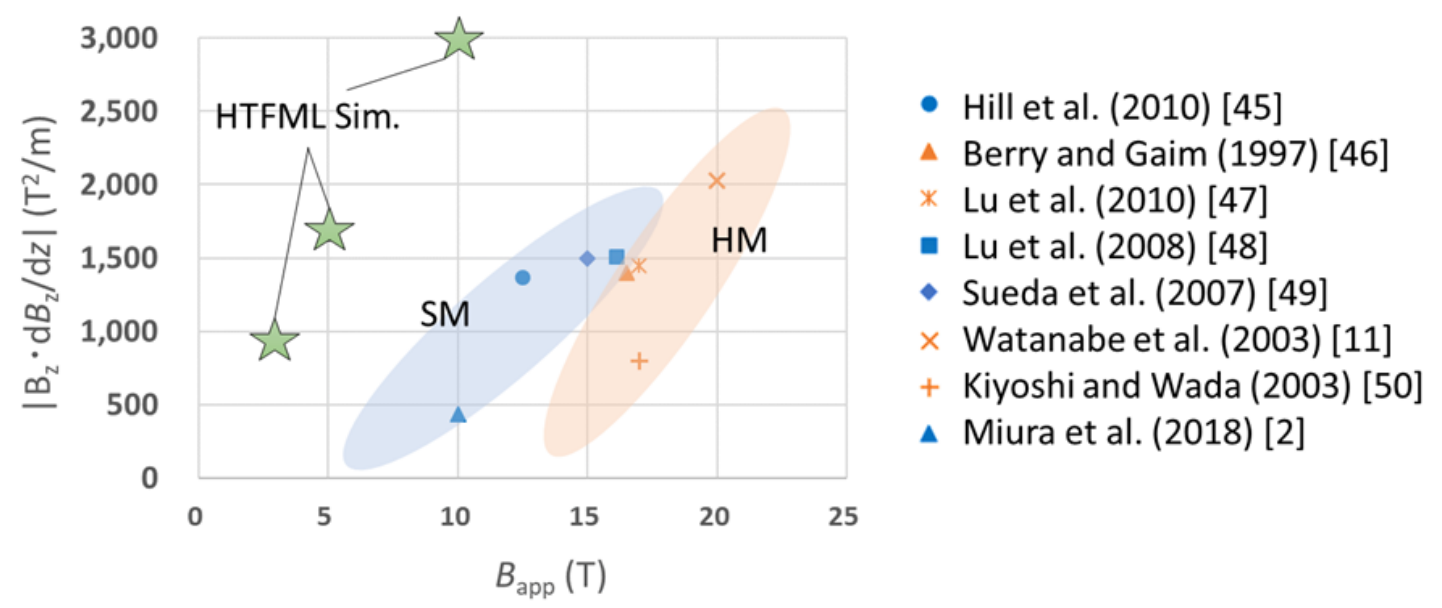

Figure 12. Relationship between the applied magnetic field, $B_{\text {app, }}$ and the magnetic field gradient product, $\left|B_{z} \cdot d B_{z} / d z\right|$, for superconducting magnets (SM) and large-scale hybrid magnet (HM) around the world $[2,11,45-50]$, which are compared to the numerical results for the HTFML. Reproduced with permission from:

[2] O Miura et al., J. Phys.: Conf. Ser. 1054012086 (2018). Copyright 2018 IOP Publishing Ltd.

[11] K Watanabe et al., Physica C 386485 (2003). Copyright 2002 Elsevier Science B.V.

[45] R J A Hill et al., Nat. Proc. 4216.2 (2010). Copyright 2010 Springer Nature.

[46] M V Berry et al., Eur. J. Phys. 18307 (1997). Copyright 1997 IOP Publishing Ltd. \& The European Physical Society.

[47] Y Liu et al., Adv. Space. Res. 45(1) 208 (2010). Copyright 2009 Elsevier Ltd.

[48] H M Lu et al., Rev. Sci. Instrum. 79(9) 093903 (2008). Copyright 2008 AIP Publishing.

[49] M Sueda et al., J. Phys. Cam. C. 111(39) 14389 (2007). Copyright 2007 American Chemical 
Society.

[50] T Kiyoshi et al., J. Low. Temp. Phys. 133(1) 31 (2003). Copyright 2003 Plenum Publishing Corporation.

\section{Conclusion}

In this study, we have proposed and analyzed a new advantage of the HTFML by numerical simulations, other than the ability to provide a continuous, concentrated magnetic field higher than the applied field reported previously: exploiting the HTFML's ultra-high magnetic field gradient product, $B_{\mathrm{z}} \cdot d B_{\mathrm{z}} / d z$, which could be highly applicable to magnetic levitation and separation as a compact and strong magnetic field source. The HTFML device consisting of a GdBaCuO TFM cylinder and $\mathrm{GdBaCuO}$ magnetic lens after magnetization from $10 \mathrm{~T}$ can generate a concentrated magnetic field, $B_{\mathrm{c}}$, of $11.4 \mathrm{~T}$ and a $B_{\mathrm{Z}} \cdot d B_{\mathrm{Z}} / d z$ over $\pm 3,000 \mathrm{~T}^{2} / \mathrm{m}$, which is higher than that of other superconducting magnets (SM) and large-scale hybrid magnets (HM) around the world. The usefulness of the HTFML device in this respect was discussed based on numerical results for magnetic separation of a mixture of precious metal particles ( $\mathrm{Pt}, \mathrm{Au}, \mathrm{Ag}$ and $\mathrm{Cu}$ ) dispersed in pure water, exploiting the magneto-Archimedes effect. The HTFML device enables the design of a compact and mobile desktop-type superconducting bulk magnet system and can improve the accessibility to strong magnetic fields in a variety of food, medical and industrial applications.

\section{Acknowledgements}

This research is supported by Adaptable and Seamless Technology transfer Program through Targetdriven R\&D (A-STEP) from Japan Science and Technology Agency (JST), Grant No. VP30218088419 and by JSPS KAKENHI Grant No. 19K05240. M. D. Ainslie would like to acknowledge financial support from an Engineering and Physical Sciences Research Council (EPSRC) Early Career Fellowship, EP/P020313/1. All data are provided in full in the results section of this paper.

\section{References}

[1] M A Weilert, D L Whitaker, H J Maris and G M Seidel Phys. Rev. Lett. 774840 (1996).

[2] O Miura, K Yamagishi and D Yamamoto J. Phys.: Conf. Ser. 1054012086 (2018).

[3] T Kobayashi, T Mori, Y Akiyama, F Mishima and S Nishijima J. Phys.: Conf. Ser. 897012018 (2017).

[4] T Oka, K Tanaka, T Kimura, D Mimura, S Fukui, J Ogawa, T Sato, M Ooizumi, K Yokoyama and M Yamaguchi Physica C 4701799 (2010). 
[5] K Takahashi, I Mogi, S Awaji, M Motokawa and K Watanabe J. Phys.: Conf. Ser. 51450 (2006).

[6] D-C Yin Prog. Cryst. Growth Charact. Mater. 611 (2015).

[7] K Hashi, S Ohki, S Matsumoto, G Nishijima, A Goto, K Deguchi, K Yamada, T Noguchi, S Sakai, M Takahashi, Y Yanagisawa, S Iguchi, T Yamazaki, H Maeda, R Tanaka, T Nemoto, H Suematsu, T Miki, K Saito and T Shimizu J. Magn. Reson. 25630 (2015).

[8] H Maeda, T Yamazaki, Y Nishiyama, M Hamada, K Hashi, T Shimizu, H Suematsu and Y Yanagisawa eMagRes 51109 (2016).

[9] Y Ikezoe, T Kaihatsu, D Sakae, H Uetake, N Hirota and K Kitazawa Energ. Convers. Manage. 43 417 (2002).

[10] V S Nikolayev, D Chatain, D Beysens and G Pichavant Microgravity. Sci. Technol. 23113 (2010).

[11] K Watanabe, K Takahashi, I Mogi, G Nishijima, S Awaji and M Motokawa Physica C 386485 (2003).

[12] Y M Liu, D M Zhu, DM Strayer and U E Israelsson Adv. Space. Res. 45208 (2010).

[13] Y Ikezoe, Y Hirota, J Nakagawa and K Kitazawa Nature 393749 (1998).

[14] J H Durrell, M D Ainslie, D Zhou, P Vanderbemden, T Bradshaw, S Speller, M Filipenko and D A Cardwell Supercond. Sci. Technol. 31103501 (2018).

[15] J H Durrell, A R Dennis, J Jaroszynski, M D Ainslie, K G B Palmer, Y-H Shi, A M Campbell, J Hull, M Strasik, E E Hellstrom and D A Cardwell Supercond. Sci. Technol. 27082001 (2014).

[16] K Huang, Y-H Shi, J Srpčič, M D Ainslie, D K Namburi, A R Dennis, D Zhou, M Boll, M Filipenko, J Jaroszynski, E E Hellstrom, D A Cardwell and J H Durrell Supercond. Sci. Technol. 33 02LT01 (2020).

[17] M D Ainslie, D Zhou, H Fujishiro, K Takahashi, Y-H Shi and J H Durrell Supercond. Sci. Technol. 29124004 (2016).

[18] D Zhou, M D Ainslie, Y Shi, A R Dennis, K Huang, J R Hull, D A Cardwell and J H Durrell Appl. Phys. Lett. 11062601 (2017).

[19] K Takahashi, M D Ainslie, H Fujishiro, T Naito, Y-H Shi and D A Cardwell Physica C 5361 (2017).

[20] H Fujishiro, T Tateiwa, A Fujiwara, T Oka and H Hayashi Physica C 334-338 445 (2006).

[21] D Zhou, M D Ainslie, J Srpčič, K Huang, Y-H Shi, A R Dennis, D A Cardwell, J H Durrell, M Boll and M Filipenko Supercond. Sci. Technol. 31105005 (2018).

[22] T Nakamura, D Tamada, Y Yanagi, Y Itoh, T Nemoto, H Utsumi and K Kose J. Magn. Reson. 25968 (2015).

[23] K Ogawa, T Nakamura, Y Terada, K Kose and T Haishi Appl. Phys. Lett. 98234101 (2011).

[24] O V Vakaliuk, M D Ainslie and B Halbedel Supercond. Sci. Technol. 31084003 (2018).

[25] C P Bean Phys. Rev. Lett. 8250 (1962).

[26] C P Bean Rev. Mod. Phys. 3631 (1962). 
[27] W Zhai, Y-H Shi, J H Durrell, A R Dennis, Z Zhang and D A Cardwell Cryst. Growth. Des. 15 907 (2015).

[28] D K Namburi, Y-H Shi, W Zhai, A R Dennis, J H Durrell and D A Cardwell Cryst. Growth. Des. 151472 (2015).

[29] ZY Zhang, S Matsumoto, S Choi, R Teranishi and T Kiyoshi Physica C 4711547 (2011).

[30] ZY Zhang, S Choi, S Matsumoto, R Teranishi, G Giunchi, A Figini Albisetti and T Kiyoshi Supercond. Sci. Technol. 25025009 (2012).

[31] ZY Zhang, S Matsumoto, R Teranishi and T Kiyoshi Supercond. Sci. Technol. 26045001 (2013).

[32] K Takahashi, H Fujishiro and M D Ainslie Supercond. Sci. Technol. 31044005 (2018).

[33] S Namba, H Fujishiro, T Naito, M D Ainslie and K Takahashi Supercond. Sci. Technol. 32 12LT03 (2019).

[34] H Fujishiro and T Naito Supercond. Sci. Technol. 23105021 (2011).

[35] H Fujishiro, M D Ainslie, K Takahashi, T Naito, Y Yanagi, Y Itoh and T Nakamura Supercond. Sci. Technol. 30085008 (2017).

[36] K Takahashi, H Fujishiro, T Naito, Y Yanagi, Y Itoh and T Nakamura Supercond. Sci. Technol. 30115006 (2017).

[37] M Li, L Li, D Xu J. Phys.: Conf. Ser. 897012016 (2017).

[38] P J Masson, M Breschi, P Tixador and C A Luongo IEEE Trans. Appl. Supercond. 171533 (2007). [39] M Jirsa, L P̊ust, D Dlouhý and M R Koblischka Phys. Rev. B 553276 (1997).

[40] T Kii, R Kinjo, N Kimura, M Shibata, M A Bakr, Y W Choi, M Omer, K Yoshida, K Ishida, T Komai, K Shimahashi, T Sonobe, H Zen, K Masuda and H Ohgaki IEEE. Trans. Appl. Supercond. B 224100904 (2012).

[41] H Fujishiro, T Naito, Y Yanagi, Y Itoh and T Nakamura Supercond. Sci. Technol. 32065001 (2019).

[42] S Namba, H Fujishiro, M D Ainslie, K Takahashi, D K Nambri, D Zhou and T Naito IEEE. Trans. Appl. Supercond. 296801605 (2019).

[43] K Watanabe, G Nishijima, S Awaji, K Takahashi, K Koyama, N Kobayashi, M Ishizuka, T Itou, T Tsurudome and J Sakurada IEEE. Trans. Appl. Supercond. 16934 (2006).

[44] T Ando, N Hirota and M Maie Sep. Prif. Technol. 149197 (2015).

[45] R J A Hill, O J Larkin, P Anthony, M R Davey, L Eaves, C E D Rees and C E Dijkstra Nat. Proc. 4216.2 (2010).

[46] M V Berry and A K Geim Eur. J. Phys. 18307 (1997).

[47] Y Liu, D M Zhu, D M Strayer and U E Israelsson Adv. Space. Res. 45(1) 208 (2010).

[48] H M Lu, D C Yin, H S Li, L Q Geng, C Y Zhang, Q Q Lu, Y Z Guo, W H Guo, P Shang and N I Wakayama Rev. Sci. Instrum. 79(9) 093903 (2008).

[49] M Sueda, A Katsuki, M Nonomura, R Kobayashi and Y Tanimoto J. Phys. Cam. C. 111(39) 
14389 (2007).

[50] T Kiyoshi and H Wada J. Low. Temp. Phys. 133(1) 31 (2003). 